\title{
Dual regulation of gene expression mediated by tetracycline and Cre recombinase
}

\author{
Jae Yoon Park ${ }^{1}$, Qing Luo ${ }^{1,2}$, Wei Jiang ${ }^{1}$, Quan Kang ${ }^{1,2}$, Ying Peng ${ }^{1}$, \\ Catherine Strom ${ }^{3}$, Hue H. Luu ${ }^{1}$, Rex C. Haydon ${ }^{1}$, and Tong-Chuan He ${ }^{1}$ \\ ${ }^{1}$ The University of Chicago Medical Center, Chicago, IL, USA, ${ }^{2}$ The Children's \\ Hospital of Chongqing University of Medical Sciences, Chongqing 400016, China, \\ and ${ }^{3}$ University of Southern California, Los Angeles, CA, USA
}

BioTechniques 36:390-396 (March 2004)

Controlled gene expression is important for elucidating gene functions and developing safer methods of gene therapy $(1,2)$. Historically, early inducible expression systems were mainly based on responses of endogenous elements to exogenous signals or stresses such as heat shock, hormones, or metal ions. However, these systems lacked specificity due to the inducer's pleiotropic effects. Consequently, nonmammalian or mutated endogenous control elements such as the lac repressor/operator, ecdysoneinducible system, Cre/Lox system, and tetracycline (Tc)-inducible system were used to achieve higher specificity of gene induction $(1,2)$. Currently, the Tc-inducible system remains as one of the most commonly used expression systems (3). The Tc-inducible system is derived from regulatory Tc-resistant determinants in Gram-negative bacteria (4). There are $11 \mathrm{Tc}$ resistance determinants, each consisting of a resistance protein (i.e., TetB) and a regulatory/ repressor protein (i.e., TetR). In the absence of Tc, TetR binds to the tet operator $\left(\right.$ tet $\left._{2}\right)$. However, the presence of Tc allows expression of Tc resistance proteins as TetR binds to Tc allosterically and dissociates from tetO $\mathrm{O}_{2}$ (4). The best understood TetR protein is a repressor for the resistance protein TetB, or TetR(B) (or TetR henceforth) (5). TetR-regulated gene expression was first shown in plant cells (6) with subsequent advances that led to the development of Tc-responsive promoters for regulated gene expression in mammalian cells $(5,7)$.

We have recently developed a single vector system, the so-called pTHE vec- tor system, in which Tc-regulated gene expression is mediated by a chimeric repressor that recruits histone deacetylases in mammalian cells (8). The chimeric repressor was engineered by fusing the TetR with an mSin3-interacting domain of human Mad1, which bound to tet $\mathrm{O}_{2}$ element with high affinity and was abrogated by doxcycline, a commonly used stable analog of Tc. This Tc-inducible system improved the previous Tc-inducible systems by using a single vector that contained both the chimeric repressor and the Tc-responsive promoter (8). However, an apparent weakness of the pTHE system is that it requires time to produce the chimeric repressor, resulting in the lack of initial repression of the gene of interest. This could be highly problematic if a gene product is growth inhibitory or toxic.

In order to improve the initial repression of transgene expression in our recently developed pTHE system (8), we inserted a LoxP-flanking stuffer containing the green fluorescent protein (GFP) coding sequence and a simian virus 40 (SV40) polyadenylation (SV40 PA) signal into the pTHE vector before the multiple linker sites and after the TetR-responsive elements (TO4) (Figure 1A). Originally identified in bacterial phage $\mathrm{P} 1$, the Cre recombinase directs specific excision of DNA flanked by two LoxP sites and reunites the LoxP sites, a 34bp sequence, consisting of two 13-bp inverted repeats separated by an 8-bp spacer region $(9,10)$. The SV40 PA element served as transcription termination to prevent possible expression of the gene of interest. Conceptually, the expression of transgene could be restored after the GFP stuffer cassette is removed by Cre recombinase. As illustrated in Figure 1A, the GFP stuffer cassette was subcloned into the EcoRI and Not I sites of the pTHE vector and positioned immediately downstream of the TetR binding sites. The resulting vector was designated as pTLOX. In order to test the functionality of this vector, we subcloned the coding region of firefly (Photinus pyralis) luciferase into the cloning linker of pTLOX, resulting in pTLOX-Luc (Figure 1B). To efficiently introduce Cre recombinase into mammalian cells, we constructed a recombinant adenovirus expressing the Cre recombinase (i.e., AdCRE) using our recently developed AdEasy system (11). A previously characterized adenoviral vector expressing GFP (AdGFP) was used as a control (11). Details regarding the construction of the above vectors are available upon request. A vector map and full-length sequence of pTLOX are available at http://www.boneandcancer.org/ resources.htm.

We next tested the functionality of the pTLOX vector. Using the pTLOXLuc vector, we examined the excision efficiency of the LoxP sites and restoration of luciferase expression upon the introduction of the Cre recombinase. Briefly, subconfluent HCT116 cells were transfected with pTLOX-Luc using Lipofectamine ${ }^{\mathrm{TM}}$ (Invitrogen, Carlsbad, CA, USA). At the end of transfection, cells were infected with AdCRE or AdGFP [multiplicity of infection $(\mathrm{MOI})=50$ ] $(11)$ and were incubated in complete medium without or with doxycycline $(100 \mathrm{ng} / \mathrm{mL})$. At $24 \mathrm{~h}$ after infection, cells were lysed for luciferase assays. As shown in Figure 2A, in the absence of Cre recombinase (i.e., infection with AdGFP), the luciferase activity was maintained at a basal level and was not affected by doxycycline, suggesting that the GFP stuffer cassette can effectively block the expression of transgenes. However, in the AdCREinfected cells luciferase activity was remarkably increased (Figure 2A). Specifically, the luciferase activity increased $>1000$-fold in the doxycyclineinduced and AdCRE-infected cells, whereas the luciferase activity was also elevated approximately 88 -fold in the 


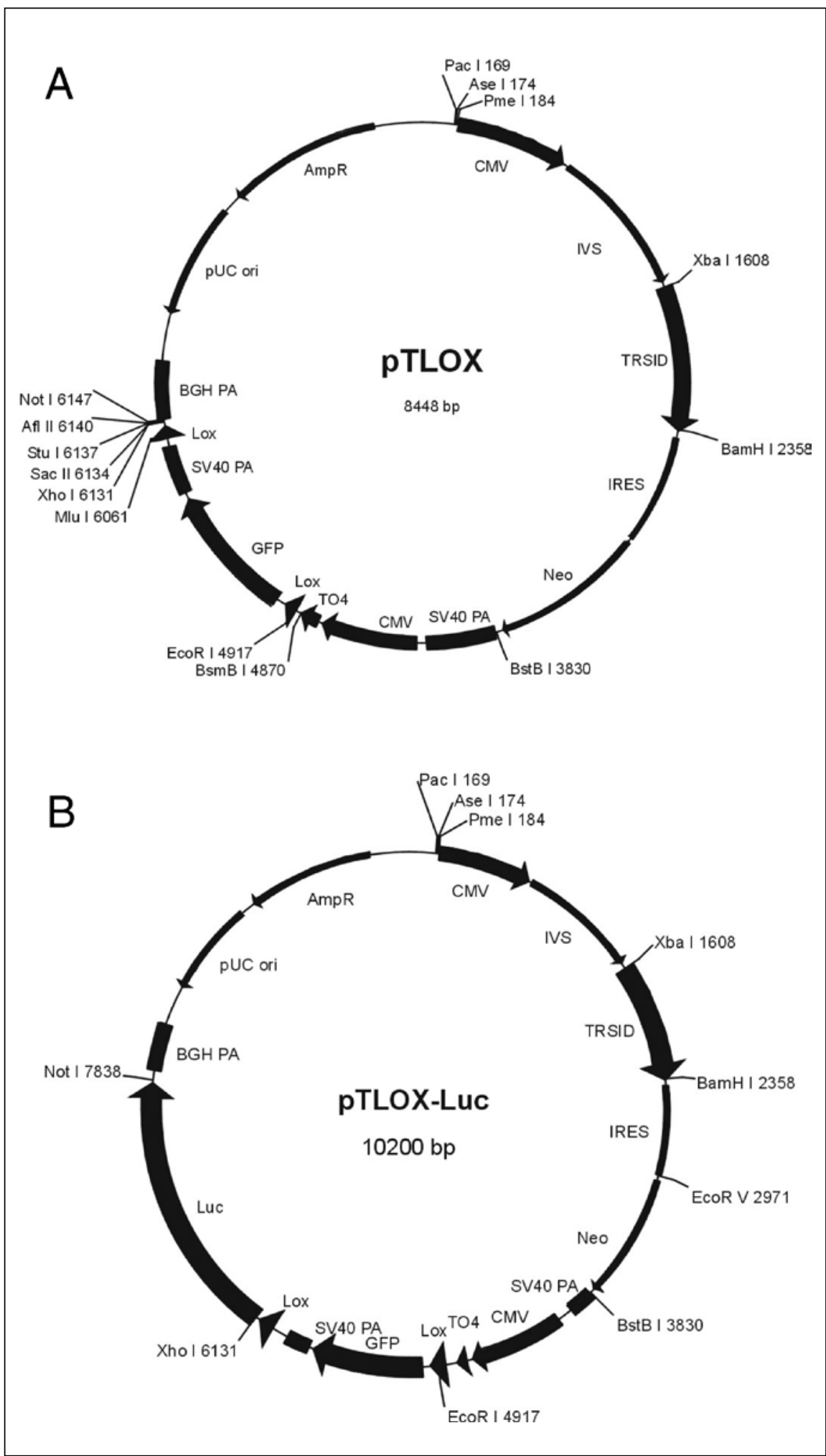

Figure 1. Representation of pTLOX and pTLOX-Luc vectors. (A) Construction of pTLOX vector. A stuffer cassette, in which the green fluorescent protein (GFP) coding region and simian virus 40 (SV40) polyadenylation element were flanked with two head-to-tail LoxP sites, was first constructed and then subcloned into the EcoRI and NotI sites of the pTHE vector, resulting in pTLOX. (B) Construction of pTLOX-Luc vector. The coding region of firefly (Photinus pyralis) luciferase gene was PCR-amplified and subcloned into the pTLOX vector at the StuI site, resulting in pTLOX-Luc. Details regarding the vector constructions are available upon request (see also Reference 8 ). 


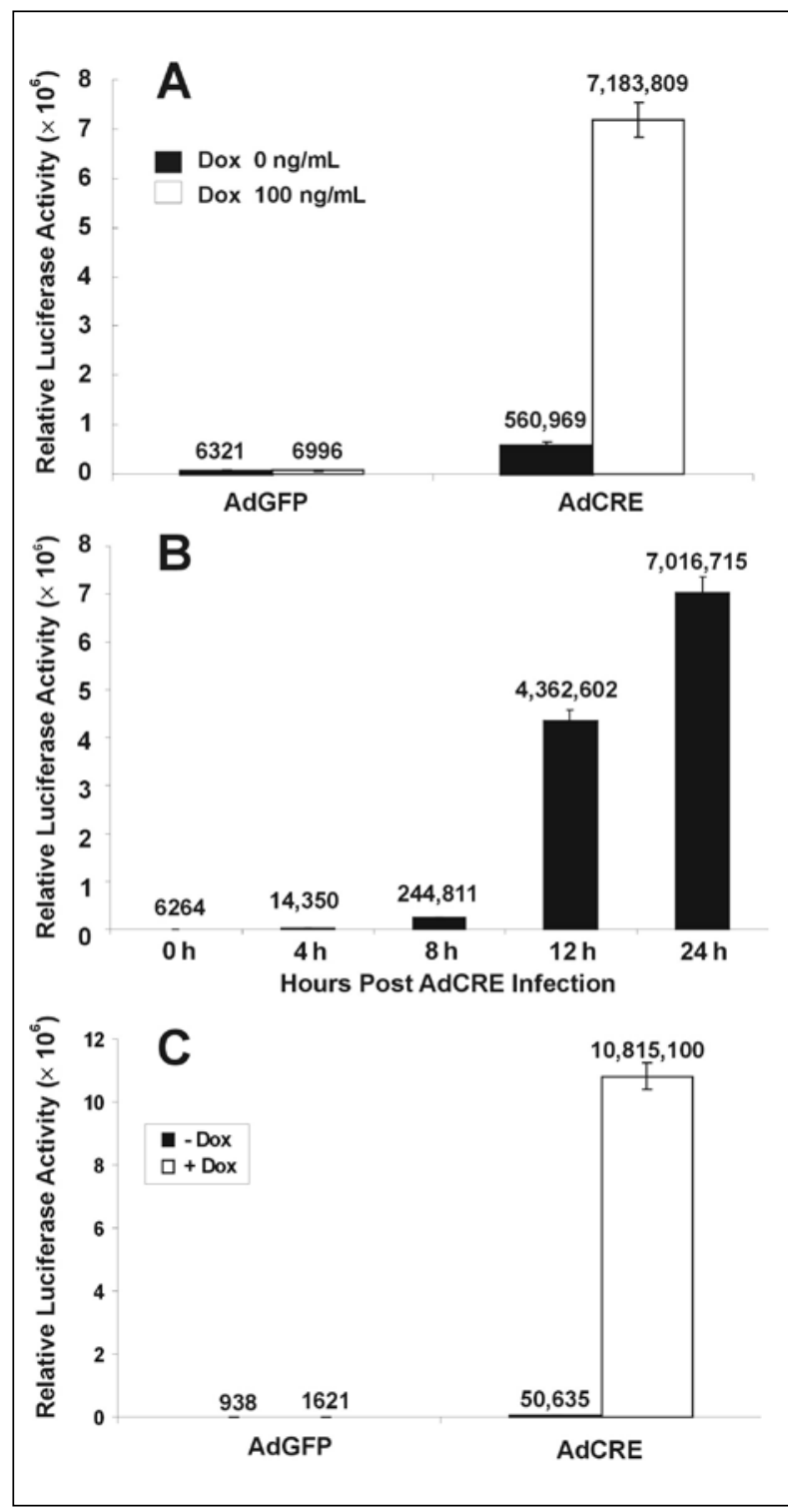

Figure 2. Dual regulation of luciferase activity by doxycycline and Cre recombinase in pTLOX-Luc. (A) Subconfluent HCT116 cells were transfected with pTLOX-Luc using Lipofectamine. At the end of transfection, cells were infected with AdCRE or AdGFP (as a control) at a predetermined optimal multiplicity of infection (MOI) of 50, and maintained in complete McCoy's 5A medium without or with 100 $\mathrm{ng} / \mathrm{mL}$ of doxycycline. At $24 \mathrm{~h}$ after infection, the cells were harvested for luciferase assays using the Luciferase Assay kit (Promega, Madison, WI, USA). (B) For the time point assays, cells were transfected with pTLOX-Luc and infected with AdCRE in the same fashion as described above and were incubated in the doxycycline-containing (100 $\mathrm{ng} / \mathrm{mL}$ ) complete medium. At 0, 4, 8, 12, and $24 \mathrm{~h}$ after infection, cells were collected for luciferase assays. Transfection efficiency was normalized by measuring the $\beta$-galactosidase activity of the co-transfected pCMV- $\beta$. (C) Subconfluent HCT116 cells were transfected with pTLOX-Luc and selected in the presence of $0.4 \mathrm{mg} / \mathrm{mL}$ for 3 weeks The stable pool was replated into 24-well plates in the absence or presence of doxycycline $(100 \mathrm{ng} / \mathrm{mL})$ and infected with AdCRE or AdGFP. Luciferase assays were carried out at $24 \mathrm{~h}$ after infection/induction. Each assay condition was carried out in triplicate, and the mean value of each treatment is shown. Representative results from at least three independent experiments are shown. CMV, cytomegalovirus; AdCRE, recombinant adenovirus expressing Cre recombinase; AdGFP, adenoviral vector expressing green fluorescent protein.
AdCRE-infected cells without doxycycline stimulation. These findings suggest that: (i) there is a lack of initial repression of transgene expression in the pTHE vector; and (ii) the pTLOX-mediated gene expression is tightly regulated by both doxycycline and Cre recombinase. Similar results were also observed in several other human and mouse cell lines (data not shown).

We sought to test the time-course restoration of luciferase expression upon the introduction of Cre recombinase. Briefly, subconfluent HCT116 cells were transfected with pTLOX-Luc and infected with AdCRE $(\mathrm{MOI}=50)$ in the presence of doxycycline
(100 ng/mL). At 0, 4, 8, 12, and $24 \mathrm{~h}$ after AdCRE infection, cells were collected for luciferase assays. As shown in Figure 2B, compared with that at 0 $h$, luciferase activity increased approximately 2-fold at $4 \mathrm{~h}, 39$-fold at $8 \mathrm{~h}$, 696-fold at $12 \mathrm{~h}$, and 1120 -fold at $24 \mathrm{~h}$, respectively. These findings have demonstrated that doxycycline and Cre recombinase can efficiently induce transgene expression in the pTLOX system. We examined the luciferase activity in longer time points (up to $96 \mathrm{~h}$ ) and found that it nearly reached the peak level at around $24 \mathrm{~h}$ (data not shown). At as early as $12 \mathrm{~h}$ after AdCRE infection, the luciferase activity reached approximately $60 \%$ of that at $24 \mathrm{~h}$, indicating that the GFP stuffer cassette was efficiently removed by the Cre recombinase expressed by the adenoviral vector. We next tested the regulation of luciferase expression in a pool of HCT116 cells stably transfected with pTLOX-Luc. As shown in Figure 2C, the introduction of both doxycycline and Cre induced an approximately 11,500-fold increase in luciferase activity. Although the luciferase activity increased approximately 50-fold in
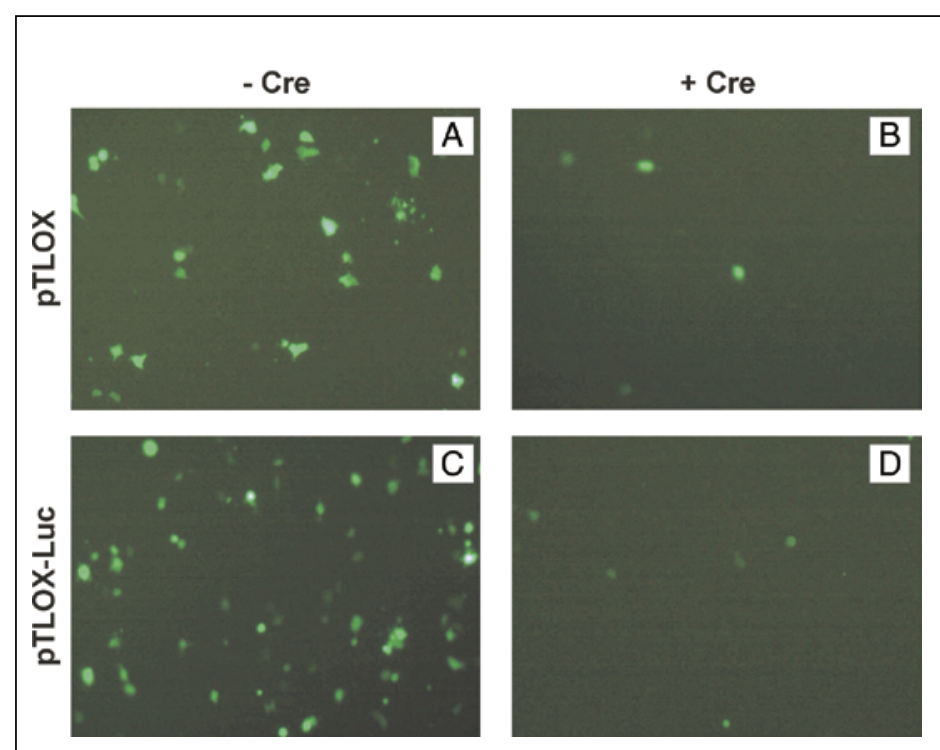

Figure 3. Cre-mediated removal of GFP expression in pTLOX and pTLOXLuc. Subconfluent HCT116 cells were transfected with pTLOX or pTLOX-Luc for $4 \mathrm{~h}$ using Lipofectamine. At the end of transfection, cells were infected with AdCRE at a multiplicity of infection (MOI) of 50, and maintained in complete McCoy's 5A medium containing $100 \mathrm{ng} / \mathrm{mL}$ of doxycycline. At $48 \mathrm{~h}$ after AdCRE infection and doxcycline induction, GFP expression was examined under a fluorescence microscope. Representative results from three independent experiments are shown. GFP, green fluorescent protein; AdCRE, recombinant adenovirus expressing Cre recombinase. 
the cells infected with AdCRE alone (i.e., no doxcycline), the basal levels in the stable pools were in general much lower than that in the transiently transfected cells (Figure 2A).

Finally, we sought to visualize the removal of the GFP stuffer cassette upon the introduction of Cre recombinase. Experimentally, subconfluent HCT116 cells were transfected with pTLOX or pTLOX-Luc. The transfected cells were induced with doxycycline $(100 \mathrm{ng} / \mathrm{mL})$ in the presence or absence of AdCRE infection. GFP expression was assessed at $48 \mathrm{~h}$ after infection. As shown in Figure 3, GFP signal was readily detectable in the absence of $\mathrm{Cre}$ recombinase expression (Figure 3, A and $\mathrm{C}$ ). However, a marked decrease in GFP signal was observed in the cells that were infected with AdCre (Figure 3 , B and D), indicating that the GFP stuffer cassette was efficiently excised by the Cre recombinase expressed by the recombinant adenoviral vector. Similar results have been observed in several other human and mouse cell lines, as well as in the stable pools (data not shown).

In summary, we developed and characterized a much improved inducible expression system, the so-called pTLOX vector. By exploiting the Cre recombinase-mediated site-specific recombination, the pTLOX vector overcomes the shortcomings of the lack of initial repression of transgene expression in our previously developed pTHE system (8). While retaining the advantage of the pTHE system as a singlestep Tc-inducible vector, the pTLOX vector offers additional advantages. For example, the LoxP site-flanked GFP stuffer can be used as an easy screening marker of potential stable clones since their GFP signal can be induced by doxycycline. Expression of transgenes can be further analyzed in the doxycycline-inducible GFP-expressing stable clones by introducing the Cre recombinase. Thus, the use of the GFP stuffer could significantly simplify and speed up the screening process of stable clones. A recent report described the potential utility of a LoxP-flanked stuffer for diphtheria toxin-mediated cytotoxic cancer gene therapy (12). Although the expression of the toxin was shown to be highly regulated by
Cre recombinase, the activation of gene expression was not reversible, and the level of toxin gene expression was not regulatable (12). Taken together, the pTLOX system should be useful not only for in vitro studies but also for in vivo animal studies, as transgenic animals expressing Cre recombinase or Tet regulators are readily available $(10,13-17)$.

\section{ACKNOWLEDGMENTS}

The authors wish to thank Bert Vogelstein of Johns Hopkins Oncology Center, Baltimore, $M D$, for providing HCT116 cells. This work was supported in part by research grants from the Brinson Foundation and the Schweppe Foundation. J.Y.P. was a recipient of the Howard Hughes Medical Institute Undergraduate Research Fellowship.

\section{REFERENCES}

1.Saez, E., D. No, A. West, and R.M. Evans. 1997. Inducible gene expression in mammalian cells and transgenic mice. Curr. Opin. Biotechnol. 8:608-616.

2.Rossi, F.M. and H.M. Blau. 1998. Recent advances in inducible gene expression systems. Curr. Opin. Biotechnol. 9:451-456.

3.Gossen, M. and H. Bujard. 1992. Tight control of gene expression in mammalian cells by tetracycline-responsive promoters. Proc. Natl. Acad. Sci. USA 89:5547-5551.

4.Hillen, W. and C. Berens. 1994. Mechanisms underlying expression of Tn10 encoded tetracycline resistance. Annu. Rev. Microbiol. 48:345-369.

5.Blau, H.M. and F.M. Rossi. 1999. Tet B or not tet B: advances in tetracycline-inducible gene expression. Proc. Natl. Acad. Sci. USA 96:797-799.

6.Gatz, C. and P.H. Quail. 1988. Tn10-encoded tet repressor can regulate an operatorcontaining plant promoter. Proc. Natl. Acad. Sci. USA 85:1394-1397.

7.Rossi, F.M., A.M. Kringstein, A. Spicher, O.M. Guicherit, and H.M. Blau. 2000. Transcriptional control: rheostat converted to on/off switch. Mol. Cell 6:723-728.

8.Jiang, W., L. Zhou, B. Breyer, T. Feng, H. Cheng, R. Haydon, A. Ishikawa, and T.-C. He. 2001. Tetracycline-regulated gene expression mediated by a novel chimeric repressor that recruits histone deacetylases in mammalian cells. J. Biol. Chem. 276: 45168-45174.

9.Hoess, R.H. and K. Abremski. 1984. Interaction of the bacteriophage $\mathrm{P} 1$ recombinase Cre with the recombining site loxP. Proc. Natl. Acad. Sci.USA 81:1026-1029.

10.Sauer, B. 2002. Cre/lox: one more step in the taming of the genome. Endocrine 19: 221-228.

11.He, T.C., S. Zhou, L.T. da Costa, J. Yu, K.W. Kinzler, and B. Vogelstein. 1998. A simplified system for generating recombinant adenoviruses. Proc. Natl. Acad. Sci. USA 95:2509-2514.

12.Lee, E.J. and J.L. Jameson. 2002. Cellspecific Cre-mediated activation of the diphtheria toxin gene in pituitary tumor cells: potential for cytotoxic gene therapy. Hum. Gene Ther. 13:533-542.

13.Rossant, J. and A. McMahon. 1999. "Cre"ating mouse mutants-a meeting review on conditional mouse genetics. Genes Dev. 13: 142-145.

14.Nagy, A. 2000. Cre recombinase: the universal reagent for genome tailoring. Genesis 26: 99-109.

15.Garcia, E.L. and A.A. Mills. 2002. Getting around lethality with inducible Cre-mediated excision. Semin. Cell Dev. Biol. 13:151-158.

16.Albanese, C., J. Hulit, T. Sakamaki, and R.G. Pestell. 2002. Recent advances in inducible expression in transgenic mice. Semin. Cell Dev. Biol. 13:129-141.

17.Zhu, Z., T. Zheng, C.G. Lee, R.J. Homer, and J.A. Elias. 2002. Tetracycline-controlled transcriptional regulation systems: advances and application in transgenic animal modeling. Semin. Cell Dev. Biol. 13: 121-128.

Received 5 November 2003; accepted 2 January 2004.

Address correspondence to T.-C. He, Molecular Oncology Laboratory, Department of Surgery, The University of Chicago Medical Center, 5841 South Maryland Avenue, MC3079, Chicago, IL 60637, USA. e-mail: tche@surgery.bsd.uchicago.edu 